\title{
Lysis of Protoplasts of Micrococcus lysodeikticus by Ionic Detergents
}

\author{
By A. R. GILBY* and the late A. V. FEW \\ Department of Colloid Science, University of Cambridge
}

(Received 7 December 1959)

\begin{abstract}
SUMMARY
The time course of lysis of protoplasts of Micrococcus lysodeikticus was studied by spectrophotometric methods. The lytic agents used were sodium dodecylsulphate and a range of cationic detergents which each possessed a $n-\mathrm{C}_{12}$ alkyl chain and comprised the amine hydrochloride, the trimethylammonium bromide, the pyridinium bromide and the quinolinium bromide. Also the inhibition of lysis by uranyl ions and the action of detergents on isolated protoplast membranes were investigated. It is concluded that the cationic detergents act on the complex phosphatidic acid lipid component of the protoplast membrane and that lysis results from secondary osmotic effects. Access to the reactive site by agents of similar hydrophilic-hydrophobic balance is controlled by steric factors. Anionic detergents, however, cause direct disintegration of the protoplast, membrane independent of osmotic stresses, probably by interaction with the membrane protein moiety; a concurrent interaction with lipid, possibly through a lipoprotein complex, is not definitely excluded.
\end{abstract}

\section{INTRODUCTION}

There is now a considerable body of evidence to implicate the bacterial cytoplasmic membrane as the seat of the bactericidal action of ionic detergents. This evidence was reviewed by Salton (1957) and by Pethica (1958). The differences in response as between Gram-positive and Gram-negative bacteria have been ascribed to differences in lipid content in the cell walls of the bacteria. After the initial disruption of the permeability properties of the cell membranes which is always associated with the bactericidal action, full lysis and dispersion of the structural components of the cells may follow through processes not necessarily directly connected with the detergent. In some circumstances the continuing autolytic disintegration can be inhibited by high detergent concentrations (Dawson, Lominski \& Stern, 1953). With the development of the now well-established technique of protoplast preparation by the action of lysozyme (Tomcsik \& Guex-Holzer, 1952; Weibull, 1953) it became possible to study the action of detergents directly on the protoplast membrane of some bacteria in the absence of cell wall. In earlier work (Gilby \& Few, $1957 a, b$ ) qualitative and quantitative parallels were established between the bactericidal action of detergents and their lysis of protoplasts. With the dodecyl series of detergents, the lytic action with protoplasts of Micrococcus lysodeikticus was in the sequence

$$
\mathrm{C}_{12} \mathrm{H}_{25}-\mathrm{NH}_{3}{ }^{+}>-\mathrm{N}\left(\mathrm{CH}_{3}\right)_{3}{ }^{+}>-\mathrm{SO}_{4}->-\mathrm{SO}_{3}-
$$

\footnotetext{
* Present address: Division of Entomology, C.S.I.R.O., Canberra, A.C.T., Australia.
} 
It was concluded that in both the bactericidal action and the lysis of protoplasts the site of action of ionic detergents was a lipid component of the protoplast membrane. Chemical analysis (Gilby, Few \& McQuillen, 1958) showed that the protoplast membrane of $M$. lysodeikticus contained $28 \%$ lipid which consisted mainly of a complex phosphatidic acid with properties consistent with the above interpretation. Salton (1957) investigated the action of detergents on protoplasts of Bacillus megaterium. In the present paper further investigations into the action of ionic detergents on protoplasts of $\boldsymbol{M}$. lysodeikticus and on isolated protoplast membranes will be described. A wider range of cationic detergents than previously was used to determine the importance of steric factors, and the effects of pretreatment with uranyl ions on the lysis of protoplasts by anionic and by cationic detergents were studied.

\section{METHODS}

Bacteria. Micrococcus lysodeikticus (NCTC 2665, the strain used previously) was grown on a beef tryptic-digest agar medium for $20 \mathrm{hr}$. at $37^{\circ}$. The organisms.were harvested and washed with distilled water as previously described.

Preparation of protoplasts and protoplast membranes. The procedures used were those described in earlier work (Gilby \& Few, 1957 $a, b$; Gilby et al. 1958). Protoplasts were prepared by the action on the organisms (at equiv. $10 \mathrm{mg}$. dry wt./ml.) of lysozyme $(100 \mu \mathrm{g} . / \mathrm{ml}$.) in a medium containing $\mathrm{M}$-sucrose and $0.05 \mathrm{M}-\mathrm{NaCl}$. The resulting protoplasts were centrifuged down and, after the supernatant fluid was decanted, were gently resuspended in the sucrose + sodium chloride medium. Protoplast membranes were prepared from lysozyme-induced lysates in $0 \cdot 1 \mathrm{M}-\mathrm{NaCl}$ by centrifugation at $20,000 \mathrm{~g}$ and washed in $0.05 \mathrm{M}-\mathrm{NaCl}$.

Ionic detergents. Sodium dodecylsulphate (SDS) and dodecylamine hydrochloride (DAC) were pure samples kindly supplied by Dr E. Hutchinson and Professor E. Stenhagen, respectively. Dodecyltrimethylammonium bromide (DTAB), dodecylpyridinium bromide (DPB) and dodecylquinolinium bromide (DQB) were prepared from the reaction of dodecyl bromide with the corresponding tertiary base. Pure DTAB and DPB were obtained by simply mixing the reactants followed by crystallization; the conditions for preparing and purifying DQB, a new compound, were given in Few, Gilby, Ottewill \& Parreira (1958).

Spectrophotometric determination of lysis. The optical density at wavelength $500 \mathrm{~m} \mu$ was measured at known time intervals during reaction with the detergents. The concentration of protoplasts corresponded to $0.5 \mathrm{mg}$. bacterial dry wt./ $\mathrm{ml}$., and the reaction medium ( $\mathrm{pH} 6 \cdot 3 \pm 0 \cdot 1$ ) contained $\mathrm{M}$-sucrose $+0 \cdot 05 \mathrm{M}-\mathrm{NaCl}$. The reaction temperature was $22 \pm 1^{\circ}$. In the range of protoplast and detergent concentrations used, the rate of lysis was independent of protoplast concentration. With protoplast membranes, the suspension medium was the same as for protoplasts and the membrane concentration was adjusted to give an initial optical density of $0 \cdot 25$.

Pretreatment of protoplasts with uranyl nitrate. Protoplasts to give equiv. $6 \mathrm{mg}$. dry wt./ml. were suspended in samples of the usual sucrose $+\mathrm{NaCl}$ medium which also contained uranyl nitrate at different concentrations. After $30 \mathrm{~min}$. at $20-22^{\circ}$, the protoplasts were centrifuged down, resuspended in the standard sucrose $+\mathrm{NaCl}$ medium and re-centrifuged. Finally they were resuspended in the standard medium, this stock suspension being adjusted to an optical density corresponding to 3.0 mg. wt./ml. Lysis with detergents was then carried out as above. 


\section{RESULTS}

\section{Protoplast lysis by cationic detergents}

The results of lysis with DAC and DTAB have already been published (Gilby \& Few, 1957b). The optical density/time curves for lysis with DPB and DQB are shown in Figs. 1 and 2. The general form of the lysis curves is similar with each of the four detergents; at the highest concentrations used precipitation of intracellular constituents occurred with each. This was important only for DAC since, for the other agents, lysis was complete at concentrations lower than those at which precipitation began. To compare the results, as previously (Gilby \& Few, 1957a), a standard time of lysis of $\mathbf{3 0}$ min. was adopted and the decrease in optical density

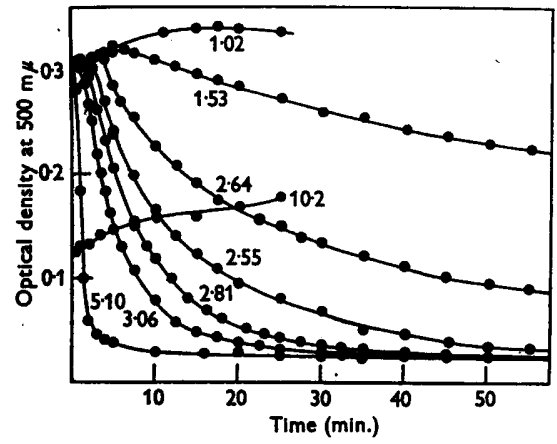

Fig. 1

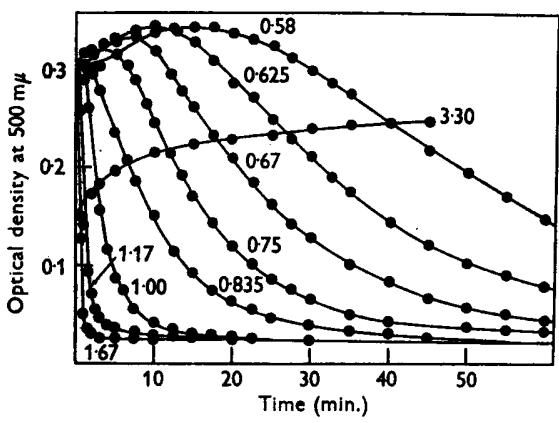

Fig. 2

Fig. 1. Lysis of Micrococcus lysodeikticus protoplasts by dodecylpyridinium bromide in $\mathrm{M}$-sucrose $+0.05 \mathrm{M}-\mathrm{NaCl}$ solution at concentrations of $\mathrm{DPB}\left(\mathrm{M} \times 10^{4}\right)$ given on curves.

Fig. 2. Lysis of protoplasts by dodecylquinolinium bromide in $\mathrm{M}$-sucrose $+0.05 \mathrm{M}-\mathrm{NaCl}$ at concentrations of DQB $\left(M+10^{4}\right)$ given on curves.

Table 1. Micrococcus lysodeikticus, protoplast lytic concentrations of cationic detergents

$\begin{array}{ccc}\text { Detergent } & \begin{array}{c}\text { Lytic concen. for } \\ \text { protoplasts } \\ \left(\mathrm{C} \times \mathbf{1 0}^{4} \mathrm{M}\right)\end{array} & \begin{array}{c}\text { Activity relative } \\ \text { to DAC, i.e. } \\ \mathrm{C}_{\text {DAC }} / \mathrm{C}_{\text {detergent }}\end{array} \\ \text { DQB } & \mathbf{0 \cdot 7 7} & \mathbf{2 \cdot 3 4} \\ \text { DAC } & 1 \cdot 8 & \mathbf{1 \cdot 0 0} \\ \text { DPB } & \mathbf{2 \cdot 6} & \mathbf{0 \cdot 6 9} \\ \text { DTAB } & \mathbf{3 \cdot 2} & \mathbf{0 . 5 6}\end{array}$

plotted against detergent concentration (Fig. 3). From each of these curves can be estimated the protoplast lytic concentration, again defined as that concentration of detergent which, in $\mathbf{3 0}$ min., decreased the optical density of a protoplast suspension by $90 \%$ of the loss observed on complete lysis; the values are given in Table 1. The protoplast lytic concentration for DAC was obtained by extrapolation of the steep curve. 


\section{Action of detergents on protoplast membranes}

The action of DTAB and of SDS on suspensions of protoplast membranes in $0.05 \mathrm{M}-\mathrm{NaCl}$ was followed by measuring changes with time of the optical density at $500 \mathrm{~m} \mu$. With DTAB, even at concentrations up to 10 times the protoplast lytic concentration, there was no change up to $12 \mathrm{hr}$. With SDS, however, the lysis curves were similar to those already published (Gilby \& Few, 1957b) for reaction of SDS with protoplasts, both in the range of SDS concentration over which reaction occurred and in the shape of the curves. Thus there was an initial rapid decrease in optical density followed by a period of at least $12 \mathrm{hr}$. during which further decrease in optical density was very slow. The extent of the initial rapid decrease in optical density depended on the SDS concentration.

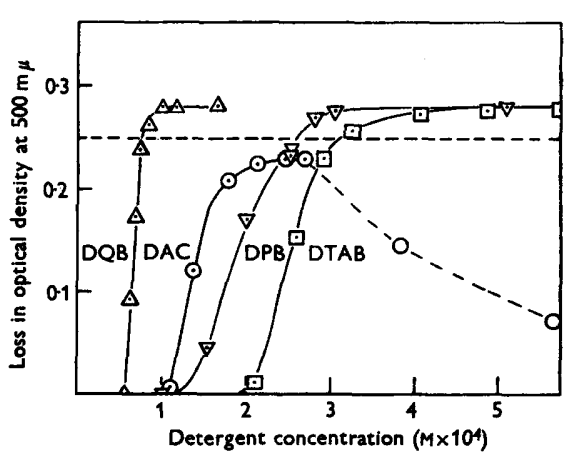

Fig. 3

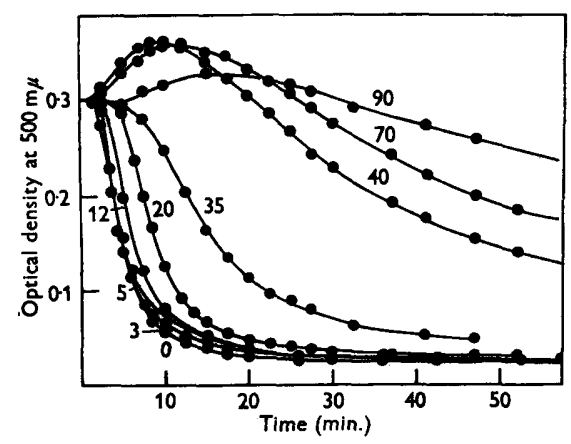

Fig. 4

Fig. 3. Decrease in optical density of protoplast suspensions after $30 \mathrm{~min}$. lysis; plotted as function of cationic detergent concentration. Broken line parallel to abscissa indicates $90 \%$ lysis.

Fig. 4. Iysis of protoplasts pretreated with uranyl nitrate by dodecyltrimethylammonium bromide in $\mathrm{M}$-sucrose $+0.05 \mathrm{M}-\mathrm{NaCl}$ solution. Concentration of DTAB $=$ $4.0 \times 10^{-4} \mathrm{M}$. Concentrations of uranyl nitrate $\left(\mathrm{M} \times 10^{5}\right)$ given on curves.

\section{Inhibition of protoplast lysis by pretreatment with uranyl nitrate}

Pretreatment of protoplasts with uranyl nitrate affected the course of lysis in a manner similar to a decrease in detergent concentration. In Fig. 4 the optical density/time curves are given for lysis by DTAB of protoplasts pretreated with uranyl nitrate at different concentrations. These curves are similar in shape to those obtained with cationic detergents and with protoplasts without uranyl ion pretreatment but where different detergent concentrations are used. The highest uranyl nitrate concentrations even induced the initial increase in optical density observed at low cationic detergent concentrations. Lysis of uranyl nitrate pretreated protoplasts with SDS gave the results shown in Fig. 5. With SDS also, the uranyl nitrate pretreatment had the same effect on the lysis/time curves as a decrease in detergent concentrations (Gilby \& Few 1957b). To demonstrate the influence on lysis of the concentration of uranyl nitrate with which protoplasts had been pretreated, the optical density at $500 \mathrm{~m} \mu$ after a standard time of $30 \mathrm{~min}$. lysis was plotted (Fig. 6) as a function of uranyl nitrate concentration. With DTAB and with SDS there was a critical range of concentration of uranyl nitrate (30-40 
$\left.\times 10^{-5} \mathrm{M}\right)$ over which there occurred a very sharp increase in the inhibition of lysis. This same critical concentration range can be shown by the application of other criteria to the results. Observations of the threshold concentration of uranyl nitrate which caused agglutination of protoplast suspensions showed that this was also in the range $30-40 \times 10^{-5} \mathrm{M}$. This critical range of uranyl nitrate concentration, important in both the lysis and coagulation of protoplasts, is considerably less than the concentration of $c .10^{-2} \mathrm{M}$ needed to reverse the charge as found by electrophoresis (Few, Gilby \& Seaman, 1960).

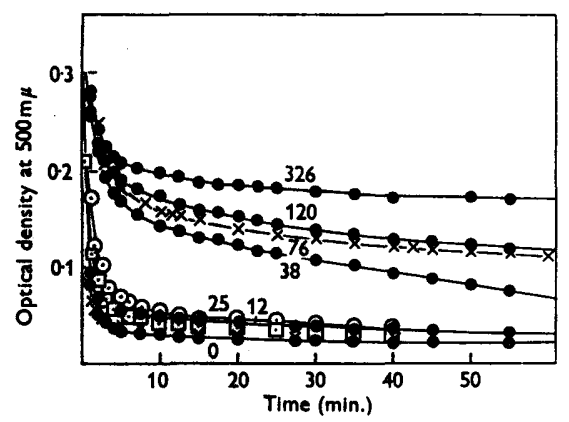

Fig. 5

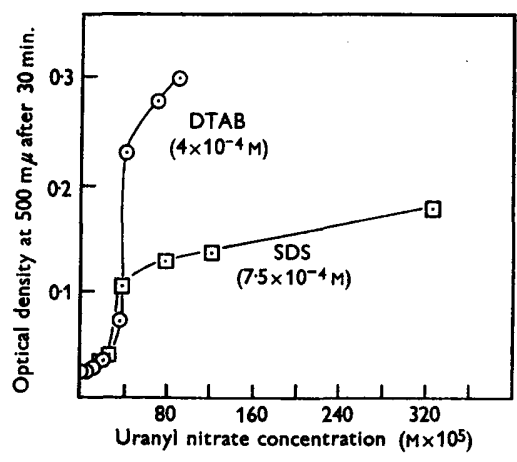

Fig. 6

Fig. 5. Lysis by sodium dodecyl sulphate of protoplasts pretreated with uranyl nitrate in M-sucrose +0.05 $\mathrm{M}$ - $\mathrm{NaCl}$ solution. Concentration of $\mathrm{SDS}=7.5 \times 10^{-4} \mathrm{M}$. Concentrations of uranyl nitrate $\left(\mathrm{M} \times 10^{5}\right)$ given on curves.

Fig. 6. Lysis by detergents of protoplasts pretreated with uranyl nitrate. Optical density after $30 \mathrm{~min}$. plotted as a function of uranyl nitrate concentration. Concentration of detergents indicated on curves.

\section{DISCUSSION}

Several factors have been considered important in governing the course of reaction of ionic detergents with the protoplast membrane of Micrococcus lysodeikticus (Gilby \& Few, $1957 b$ ), including access of ions to the reactive sites in the membrane and the interactions between polar and between non-polar groups which give rise to binding and penetration. Comparison of the relative activities of cationic detergents in protoplast lysis (summarized in Table 1) indicates the importance of the nature of the substitution in the amines used, which all possess a $\mathbf{C}_{12}$ alkyl chain, and suggests that steric factors play an important role in their interaction with protoplasts. This is strikingly demonstrated by the substituted amine, DTAB, which has an activity relative to the unsubstituted amine of only 0.56. Inspection of molecular models of the detergents shows that the reactivity sequence DAC > DPB > DTAB is the opposite of the sequence of relative size of the ionizable head groups. Thus the most bulky group, $-\mathbf{N}\left(\mathrm{CH}_{3}\right)_{3}{ }^{+}$, had the lowest lytic activity. This might be due to diminished ion-dipole forces (proportional to $Z / r^{3}$ ) involved in adsorption of the detergent, or possibly to restricted access to an internal adsorption site. Contrary to its actual ranking as the most active agent studied, DQB would, from size considerations, be expected to be less active than DPB. This enhanced activity seems likely to be due to the additional benzene ring of the quinolinium system which could cause a shift in the hydrophylic-hydrophobic 
balance of the molecule and thus alter its adsorption and penetrative powers. Analysis of the DQB used showed that impurities, which might have increased its activity, were absent (Few et al. 1958).

The protoplast membrane of Micrococcus lysodeikticus is a complex structure of protein, lipid and carbohydrate (Gilby et al. 1958). The lipid component consists chiefly of a complex phosphatidic acid and occupies an internal site in the membrane (Few et al. 1960). Such a phospholipid would provide suitable ionic groups for electrostatic interaction with cationic detergents. Furthermore, the net negative charge at the surface of the protoplast would favour the adsorption of cationic detergent ions. The inhibition of lysis by pretreatment with uranyl ions suggests that the important site of action in the protoplast membrane may be the phosphate groups of the membrane lipid. Some workers (e.g. Massart, 1952) have described a decrease in toxicity of surface-active cations by competing cations. Newton (1954) postulated that the extreme effectiveness of uranyl ions in protecting Pseudomonas aeruginosa from the lytic action of polymyxin suggested that the polymyxincombining loci of that cell surface may be polyphosphates. The phosphate groups of the membrane lipid of $M$. lysodeikticus are particularly sensitive to uranyl ions (Few et al. 1960). As stressed previously (Gilby \& Few, 1957b) DTAB is not bound by proteins such as bovine plasma albumin at concentrations which produce rapid lysis of protoplasts (Few, Ottewill \& Parreira, 1955). It is therefore concluded that, in protoplast lysis, cationic detergents react with the phosphatidic acid lipid component of the protoplast membrane to disrupt its permeability properties. With detergents of similar hydrophilic-hydrophobic balance, the relative activity is controlled by the size of head group which can effect accessibility of the cationic detergent to the internal lipid site as well as the initial adsorption.

In previous work (Gilby \& Few, 1957b) no distinction was made between the action of cationic and anionic detergents, both of which were assumed to lyse by essentially the same type of action. However, inspection of the lysis/time curves reveals a marked difference between the curves obtained with each type of detergent. Whereas the cationic detergents decreased the optical density to that of complete lysis at a rate depending on detergent concentration, the anionic detergents caused a comparatively rapid initial decrease to an optical density which subsequently changed only very slightly over many hours. No detailed picture of the mechanism of lysis has been established to explain the nature of the lysis/time curves for anionic detergents or the condition of the protoplasts which survive the initial rapid lysis. In the stationary state, when either more detergent or more protoplasts are added, the change in optical density is resumed and a new stationary value reached. This suggests that this behaviour reflects the distribution of susceptibility to anionic detergents among the protoplasts. However, it is possible to invoke other ad hoc ideas to explain such observations. For example it was postulated for erythrocytes by Love (1950) that anionic detergents exert a protective as well as a lytic action.

With suspensions of protoplast membranes of Micrococcus lysodeikticus, which are not subject to any osmotic stress, SDS had a precisely similar reaction curve to that with protoplasts, while DTAB appeared to have no action on the isolated membranes. Salton (1957) noted similar differences in the membrane residues visible by phase microscopy after lysis of protoplasts of Bacillus megaterium by 
detergents. Whereas SDS appeared completely to disperse the protoplast membranes, lysis of protoplasts of $\boldsymbol{B}$. megaterium by cetyl trimethylammonium bromide was not accompanied by dispersion of the protoplast membranes. The differences in the form of the reaction curves of SDS and of DTAB with protoplast membranes of $M$. lysodeikticus suggest that a difference in action between the two types of detergent also exists in the reactions with isolated membranes, as well as in protoplast lysis. SDS interacts much more strongly with protein than does DTAB (Few et al. 1955). It therefore seems likely that the action of anionic detergents on the isolated protoplast membrane of $\boldsymbol{M}$. lysodeikticus involves the protein moiety of the membrane and results in complete dissolution of the membrane.

Since in the protoplast-lysis experiments the concentration of membranes which might result from lysis was so low that the membranes could scarcely affect the measured optical densities, the identical nature of the protoplast and the membrane lysis curves suggests that a similar mechanism may operate in both protoplast lysis and membrane disintegration by anionic detergents. In this case, protoplast lysis would depend on the dissolution of protoplast membrane and not on the disruption of the membrane's impermeability to the stabilizing sucrose, then followed by osmotic explosion. On the other hand, it may be argued that the similarity of the concentration ranges for DTAB and for SDS in which uranyl nitrate inhibited protoplast lysis (Fig. 6) suggests a similar site of action for both detergents. It seems possible that SDS may react with both protein and lipid, or alternatively with a combination of both as lipoprotein. Van der Waals forces and ion-dipole interactions, for example with hydroxyl groups in the membrane lipid shown to be present by infrared spectroscopy (Gilby $\&$ Few, 1957b), might well be sufficient to cause adsorption and penetration of the lipid by SDS. A similar concept of two concurrent mechanisms which act in the haemolytic action of anionic detergents on red cells was suggested by Rideal \& Taylor (1957). However, such an argument may not be valid for the lysis of protoplasts. It seems certain that anionic and cationic detergents act on different sites in the membrane or else act on a similar site but in different ways. From the evidence presented here it is concluded that the crucial action of anionic detergents in the lysis of protoplasts of Micrococcus lysodeikticus is with a protein component of the protoplast membrane, which might be involved in a lipoprotein complex; the direct result of this action is dissolution of the membrane. Cationic detergents, however, cause lysis as a secondary result of their action on the membrane lipid.

We thank Dr J. H. Schulman for helpful discussions and the Commonwealth Scientific and Industrial Reseach Organization, Australia, for a Postgraduate Overseas Studentship (A. R. G.). The late A. V. Few was a member of the scientific staff of the Medical Research Council.

\section{REFERENCES}

Dawson, I. M., Lominski, I. \& Stern, H. (1953). An electron-microscope study of the action of cetyl-trimethylammonium bromide on Staphylococcus aureus. J. Path. Bact. 66, 513.

Few, A. V., Girby, A. R., Ottewill, R. H. \& Parreira, H. C. (1958). Dodecylquinolinium bromide. J. chem. Soc. p. 1712 . 
Few, A. V., Grlby, A. R. \& Seaman, G. V. F. (1960). An electrophoretic study on structural components of Micrococcus lysodeikticus. Biochim. biophys. Acta, 38, 130.

Few, A. V., OTrewill, R. H. \& Parreira, H. C. (1955). The interaction between bovine plasma albumin and dodecyltrimethylammonium bromide. Biochim. biophys. Acta, 18, 136.

Gruby, A. R. \& FEw, A. V. $(1957 a)$. Reactivity of ionic detergents with Micrococcus lysodeikticus. Nature, Lond. 179, 422.

Gilby, A. R. \& FEw, A. V. (1957b). Surface chemical studies on the protoplast membrane of Micrococcus lysodeikticus. Proc. 2nd int. Congr. Surface Activity, 4, 262.

Gilby, A. R., Few, A. V. \& McQurluse, K. (1958). The chemical composition of the protoplast membrane of Micrococcus lysodeikticus. Biochim. biophys. Acta, 29, 21.

Love, L. H. (1950). The haemolysis of human erythrocytes by sodium dodecyl sulphate. J. cell. comp. Physiol. 36, 133.

Massart, L. (1952). Substances chimothérapeutiques cationiques et compétitions entre cations. Bull. Soc. Chim. biol., Paris, 34, 1145.

Newton, B. A. (1954). Site of action of polymyxin on Pseudomonas aeruginosa: Antagonism by cations. J. gen. Microbiol. $10,491$.

Pethica, B. A. (1958). Lysis by physical and chemical methods. J. gen. Microbiol. 18, 473.

Rideal, E. K. \& Taylor, F. H. (1957). On haemolysis by anionic detergents. Proc. roy. Soc. B, 146, 225.

Salton, M. R. J. (1957). The action of lytic agents on the surface structures of the bacterial cell. Proc. 2nd int. Congr. Surface Activity, 4, 245.

Tomcsik, J. \& GuEx-Holzer, S. (1952). Änderung der Struktur der Bakterienzelle im Verlauf der Lysozym-Einwirkung. Schweiz. Z. allg. Path. 15, 518.

Werbuld, C. (1953). The isolation of protoplasts from Bacillus megaterium by controlled treatment with lysozyme. J. Bact. 66, 688. 